\title{
SPECTRAL VANISHING VISCOSITY METHOD FOR NONLINEAR CONSERVATION LAWS*
}

\author{
BEN-YU GUO ${ }^{\dagger}$, HE-PING MA MAND EITAN TADMOR $^{\S}$
}

\begin{abstract}
We propose a new spectral viscosity (SV) scheme for the accurate solution of nonlinear conservation laws. It is proved that the SV solution converges to the unique entropy solution under appropriate reasonable conditions. The proposed SV scheme is implemented directly on high modes of the computed solution. This should be compared with the original nonperiodic SV scheme introduced by Maday, Ould Kaber, and Tadmor in [SIAM J. Numer. Anal., 30 (1993), 321-342], where SV is activated on the derivative of the SV solution. The new proposed SV method could be viewed as a correction of the former, and it offers an improvement which is confirmed by our numerical experiments. A postprocessing method is implemented to greatly enhance the accuracy of the computed SV solution. The numerical results show the efficiency of the new method.
\end{abstract}

Key words. spectral method, vanishing viscosity, conservation law

AMS subject classifications. 65M70, 35L65, 35L50

PII. S0036142999362687

1. Introduction. Spectral methods employ various orthogonal systems of infinitely differentiable functions to represent an approximate projection of the exact solution sought. The resulting high accuracy of spectral algorithms was a major motivation behind their rapid development in the past three decades, e.g., see Gottlieb and Orszag [11], Canuto et al. [4], Bernardi and Maday [3], and Guo [15]. The high accuracy of the spectral algorithm hinges on the global smoothness of the underlying solution.

Here we discuss spectral approximations to nonlinear conservation laws whose solutions may develop spontaneous jump discontinuities, i.e., shock waves. In this context, "physically relevant" entropy solutions must be admitted. Due to the presence of shock discontinuities, spectral approximations of entropy solutions experience spurious Gibbs oscillations, which in turn lead to two related difficulties: loss of accuracy in the overall computational domain and, in the nonlinear case, loss of instabilities. To solve both difficulties, the spectral viscosity (SV) method was introduced by Tadmor [29] in the context of Fourier approximation to nonlinear conservation laws. The main ingredient of the SV method is the use of high-frequencies diffusion which stabilizes the spectral computation without sacrificing spectral accuracy. Further results on the periodic SV method can be found in $[22,30,26,32,6]$. A more robust periodic (hyper-)SV based on hyperdiffusion of high frequencies was introduced in [33]. Maday, Ould Kaber, and Tadmor [23] were the first to consider the nonperiodic Legendre pseudospectral viscosity method for an initial-boundary value problem, and

${ }^{*}$ Received by the editors October 11, 1999; accepted for publication (in revised form) April 25, 2001; published electronically September 19, 2001.

http://www.siam.org/journals/sinum/39-4/36268.html

†School of Mathematical Sciences, Shanghai Normal University, Shanghai, China 200234 (byguo@guomai.sh.cn). This author's work was supported by the China State Major Key Project for Basic Research N. G1999032804 and by Shanghai National Science Foundation N. 00JC14057.

${ }^{\ddagger}$ Department of Mathematics, Shanghai University, Shanghai, China 200436 (hpma@guomai.sh.cn). This author's work was supported by the China State Major Key Project for Basic Research N. G1999032804.

$\S$ Department of Mathematics, University of California at Los Angeles, Los Angeles, CA 90095 (tadmor@math.ucla.edu). This author's work was supported by NSF grant DMS01-07428 and ONR grant N00014-91-J-1076. 
Ma [20, 21] recently developed the nonperiodic Chebyshev-Legendre approximation, based on the ideas of hyper-SV. For recent applications consult $[2,17,19,25,8]$.

In this paper we propose a new form for the nonperiodic SV method. The proposed SV, presented in section 2, is implemented directly on high modes of the computed solution. In the original nonperiodic SV scheme, Maday, Ould Kaber, and Tadmor [23] advocated an SV which is activated on the derivative of the SV solution. Here we point out a correction to [23]. Indeed, compared with the SV operator in [23], the correction proposed here offers an improvement which is confirmed by the numerical result in section 4 .

The question of convergence addressed in section 3 below deals with the second difficulty of spectral methods mentioned above, the issue of stability. We conclude our introduction by referring to the first difficulty regarding loss of accuracy in the presence of shock discontinuities. As we argued before [23, section 2.1], the SV solution should be viewed as a more faithful approximation to the projection of the exact solution, rather than the solution itself. The computations of Shu and Wong in [27] confirm the high accuracy of the computed SV solution as an approximation to the appropriate projection of the exact solution. The spectral content of SV solutions in the context of propagating singularities in linear transport equations was proved in Abarbanel, Gottlieb, and Tadmor [1]. Thus, the convergence rate of the SV solution is limited to the first-order convergence rate of the oscillatory exact projections. To accelerate their convergence, thus recovering the full content of the exact solution with spectral accuracy, one needs to postprocess the SV solution at its final stage. Such postprocessing filters were devised in [14, 24] away from the edges of the shocks; consult [23, section 2.1] for the nonperiodic framework and $[13,12]$ for postprocessing up to the shocks. For a recent study that combines an effective edge detector with spectral postprocessing we refer to $[7,8,9]$. In section 4 we use the Gegenbauer polynomial partial sum advocated in [12] to postprocess the SV solution so that spectral accuracy can be recovered.

\section{The SV scheme.}

2.1. Entropy solutions of nonlinear conservation laws. We consider the nonlinear scalar conservation law over the finite interval $\Lambda:=(-1,1)$,

$$
\partial_{t} u(x, t)+\partial_{x} f(u(x, t))=0, \quad(x, t) \in \Lambda \times[0, T],
$$

with $H_{\text {loc }}^{1}[0, T]$ boundary values prescribed at the inflow boundary points along $\{ \pm 1\} \times$ $[0, T]$

$$
u( \pm 1, t)=g_{ \pm}(t), \quad \pm f^{\prime}(u( \pm 1, t))<0, \quad t>0,
$$

and subject to $H^{1}(\Lambda)$-initial conditions given at $t=0$,

$$
u(x, 0)=u_{0}(x), \quad x \in(-1,1), \quad x \in\{ \pm 1\} .
$$

An entropy weak solution of (2.1) is sought, i.e., a bounded measurable $u(x, t)$, which assumes the prescribed initial and boundary data in the proper sense and admits the following entropy condition: For all convex entropy pairs $(U, F), \quad U^{\prime \prime}(\cdot) \geq 0$ satisfying the compatibility relation $F^{\prime}(\cdot)=U^{\prime}(\cdot) f^{\prime}(\cdot)$, there holds

$$
\partial_{t} U(u(x, t))+\partial_{x} F(u(x, t)) \leq 0, \quad(x, t) \in \Lambda \times[0, T] .
$$

The entropy inequality (2.4) is sufficient, in the scalar case, to single out a unique, physically relevant solution. This so-called entropy solution could be realized by the 
vanishing viscosity limit, $u=\lim _{\varepsilon} u^{\varepsilon}(x, t)$, where $u^{\varepsilon}$ satisfies the regularized vanishing viscosity equation

$$
\partial_{t} u^{\varepsilon}(x, t)+\partial_{x} f\left(u^{\varepsilon}(x, t)\right)=\varepsilon \partial_{x}\left(D \partial_{x} u^{\varepsilon}(x, t)\right), \quad D>0 .
$$

We note in passing that the regularized viscosity equation admits an equivalent weak formulation, namely, for all $\phi \in C_{0}^{\infty}\left(\Lambda \times \mathbb{R}_{t}^{+}\right)$

$$
\int_{\Lambda \times \mathbb{R}_{t}^{+}} u^{\varepsilon}(x, t) \partial_{t} \phi(x, t)+f\left(u^{\varepsilon}(x, t)\right) \partial_{x} \phi(x, t)+\varepsilon D \partial_{x} u^{\varepsilon}(x, t) \partial_{x} \phi(x, t) d x d t=0 .
$$

For the classical theory of such entropy solutions we refer to Lax [18] and Smoller [28]. For the corresponding theory of initial-boundary value problems, cf. [16] and the references therein. Tartar [34] introduced compensated compactness arguments to study the existence and stability of such solutions. In this context, one seeks a sequence of approximate weak solutions with entropy production compact in $H_{\text {loc }}^{-1}(\Lambda \times[0, T])$; an $L^{\infty}$ weak-star convergence of the corresponding fluxes then follows. Following Tadmor in [29, 23], we shall use compensated compactness arguments to answer the stability question of the SV method discussed in this paper.

2.2. The discrete framework. We let $\mathbb{P}_{N}$ denote the space of algebraic polynomials of degree $\leq N$, and we let $\left(L_{k}\right)_{k \geq 0}$ denote the orthogonal family of Legendre polynomials in this space

$$
\left(L_{j}, L_{k}\right)=\frac{2}{2 k+1} \delta_{j k}
$$

Here, $(\cdot, \cdot)$ and $\|\cdot\|$ represent the usual $L^{2}(\Lambda)$-inner product and norm. Next we let $\left\{\xi_{j}\right\}_{j=0}^{N}$ denote the zeros of $\left(1-x^{2}\right) L_{N}^{\prime}(x)$ with $\xi_{0}=-1<\xi_{1}<\cdots<\xi_{N}=1$. In what follows we shall use the Legendre Gauss-Lobatto quadrature rule, stating that there exist weights, $\omega_{j}$, such that for all $\phi \in \mathbb{P}_{2 N-1}(\Lambda)$ we have (see, e.g., [4])

$$
\int_{-1}^{1} \phi(x) d x=\sum_{j=0}^{N} \omega_{j} \phi\left(\xi_{j}\right) .
$$

This suggests that we define a discrete inner product $(., .)_{N}$ as

$$
(\phi, \psi)_{N}=\sum_{j=0}^{N} \omega_{j} \phi\left(\xi_{j}\right) \psi\left(\xi_{j}\right),
$$

and we let $\|\cdot\|_{N}$ denote the corresponding discrete norm. Indeed, this discrete norm is equivalent to the usual $L^{2}$-norm over $\mathbb{P}_{N}(\Lambda)$ :

$$
\|\phi\| \leq\|\phi\|_{N} \leq \sqrt{2+\frac{1}{N}}\|\phi\| \quad \forall \phi \in \mathbb{P}_{N}
$$

and, of course, due to (2.6) we obtain

$$
(\phi, \psi)=(\phi, \psi)_{N} \quad \text { if } \operatorname{deg} \phi+\operatorname{deg} \psi \leq 2 N-1 .
$$


Associated with the $N+1$ points of the Legendre Gauss-Lobatto quadrature rule, $\left\{\xi_{j}\right\}_{j=0}^{N}$, is a unique $\mathbb{P}_{N}$-interpolant which we denote by $\mathcal{I}_{N}$ :

$$
\mathcal{I}_{N}(\phi)(x) \equiv \sum_{k=0}^{N} \frac{\left(\phi, L_{k}\right)_{N}}{\left\|L_{k}\right\|_{N}^{2}} L_{k}(x), \quad \mathcal{I}_{N}(\phi)\left(\xi_{j}\right)=\phi\left(\xi_{j}\right), \quad j=0,1, \ldots, N .
$$

The projection $\mathcal{I}_{N}$ can be viewed as an "approximate identity" in the $\mathbb{P}_{N}$-space; in this context we recall the result of [3] which provides us with the estimate

$$
\left\|\frac{\partial}{\partial x} \mathcal{I}_{N} \phi\right\|+N \cdot\left\|\phi-\mathcal{I}_{N} \phi\right\| \leq C\left\|\frac{\partial}{\partial x} \phi\right\| .
$$

We note in passing that similar estimates hold for some other "approximate identities"

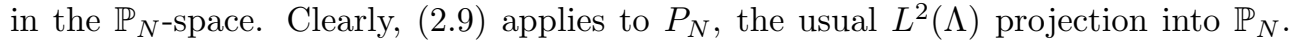
For instance, (2.9) remains valid if we replace $\mathcal{I}_{N} \phi$ with $\mathcal{J}_{N} \phi$ :

$$
\mathcal{J}_{N} \phi:=\int_{-1}^{x} P_{N-1} \frac{\partial}{\partial x} \phi d x .
$$

Indeed, using standard estimates of the latter (consult [4]), we obtain

$$
\left\|\frac{\partial}{\partial x} \mathcal{J}_{N} \phi\right\|+N \cdot\left\|\phi-\mathcal{J}_{N} \phi\right\| \leq C\left\|\frac{\partial}{\partial x} \phi\right\| .
$$

Finally, using (2.8) with $\psi \equiv \mathcal{I}_{N-1} \psi+\left(\psi-\mathcal{I}_{N-1} \psi\right)$ followed by $(2.9)$ implies that the error of Gauss quadrature for $\mathbb{P}_{2 N}$-polynomials does not exceed

$$
\left|(\phi, \psi)-(\phi, \psi)_{N}\right| \leq C\left\|\psi-\mathcal{I}_{N-1} \psi\right\|\|\phi\| \leq \frac{C}{N}\left\|\partial_{x} \psi\right\| \cdot\|\phi\| \quad \forall \phi, \psi \in \mathbb{P}_{N}(\Lambda) .
$$

2.3. The SV scheme. We seek an $N$-degree approximate solution, $u_{N}(x, t)$, which approximates the interpolant of the exact entropy solution, $\mathcal{I}_{N} u(x, t)$. Initially, we set $u_{N}(x, 0)=\mathcal{I}_{N} U_{0}(x)$. To evolve in time, we introduce the following SV operator, $Q$. Expressed in terms of the Legendre expansion $v=\sum_{l=0}^{\infty} \hat{v}_{l} L_{l}$, the SV operator, $Q$, takes the form

$$
Q v(x):=\sum_{l=0}^{N} \hat{q}_{l} \hat{v}_{l} L_{l}(x), \quad v=\sum_{l=0}^{\infty} \hat{v}_{l} L_{l}(x) .
$$

Here, $\hat{q}_{l}$ are the so-called viscosity coefficients,

$$
\begin{cases}\hat{q}_{l}=0 & \text { for } l \leq m, \\ \hat{q}_{l} \geq 1-\frac{m^{2}}{l^{2}} & \text { for } m<l \leq N,\end{cases}
$$

which are at our disposal. Observe that the SV operator is activated by only the high mode numbers, $\geq m$. In particular, if we let $m \uparrow \infty$, then the SV operator is spectrally small (in the sense that $\|Q v\|_{H^{-s}} \leq \mathrm{cm}^{-s}\|v\|$ ). We shall occasionally highlight the dependence of the SV operator on this cut off of high wave numbers, writing $Q=Q_{m}$. 
Equipped with the SV operator (2.12)-(2.13), we now construct our Legendre viscosity approximation of the initial-boundary value problem (2.1). To this end we let $u_{N}(x, t) \in \mathbb{P}_{N}$ for $t \geq 0$ be determined by the moment condition-a discrete analogue of the weak formulation (2.5) requiring that for all $\phi \in \mathbb{P}_{N}, t>0$, we have

$$
\begin{array}{r}
\left(\partial_{t} u_{N}(t)+\partial_{x} \mathcal{I}_{N} f\left(u_{N}(t)\right), \phi\right)_{N}+\varepsilon_{N}\left(\partial_{x} Q u_{N}(t), \partial_{x} Q \phi\right)_{N} \\
=\left(B\left(u_{N}(t)\right), \phi\right)_{N} \quad \forall \phi \in \mathbb{P}_{N} .
\end{array}
$$

Here, $B(\cdot)$ is a penalty boundary operator,

$$
B\left(u_{N}(t)\right)=(\lambda(t)(1-x)+\mu(t)(1+x)) \partial_{x} L_{N}(x),
$$

where the free pair of "Lagrange-multipliers," $(\lambda, \mu)$, are chosen to match the inflow boundary data, $u_{N}(x, t)=g_{ \pm}(t)$, prescribed at $x=1$ whenever $f^{\prime}\left(u_{N}(1, t)\right)<0$ and at $x=-1$ whenever $f^{\prime}\left(u_{N}(-1, t)\right)>0$. Consult [10] for the corresponding formulation of the linear stability theory.

The SV method depends on two free parameters: the vanishing amplitude of the viscosity $\varepsilon=\varepsilon_{N}$ and the size of the viscosity-free spectrum $m=m_{N}$. As in [23], we choose

$$
\varepsilon=\varepsilon_{N} \sim c N^{-\alpha}, \quad m=m_{N} \sim c N^{\beta}, \quad 0<4 \beta<\alpha \leq 1 .
$$

In particular, an increasing portion of the spectrum of size $m_{N} \sim N^{\beta}$ remains viscous free, thus retaining the (formal) spectral accuracy of the SV scheme (2.14) with the underlying conservation law (2.1).

Remark. We do not claim the parameterization in (2.15) to be optimal. In particular, arguing along the lines of $[33,20,21]$, one can use hyperviscosity regularization to increase the size of the viscosity-free modes, $m_{N}$, thus obtaining better resolution of the resulting SV scheme.

We close this section by explaining how the SV method (2.14) can be implemented as a collocation method. We first realize the $\mathrm{SV}$ in terms of an $N$-degree polynomial, $\vee_{N}$, such that

$$
\left(\partial_{x}\left(Q u_{N}\right), \partial_{x}(Q \phi)\right)_{N}=\left(\vee_{N}, \phi\right)_{N} \quad \forall \phi \in \mathbb{P}_{N}
$$

Recall that the discrete inner product $(\cdot, \cdot)_{N}$ involves the Gauss-Lobatto weights, $W:=\operatorname{diag}\left(\omega_{0}, \ldots, \omega_{N}\right)$. If we let $D_{Q}$ denote the $(N+1) \times(N+1)$ differentiation matrix associated with the derivative of the SV so that $\left(D_{Q} \phi\right)\left(\xi_{j}\right)=\partial_{x}(Q \phi)\left(\xi_{j}\right)$, then $0 \leq$ $j \leq N$ for all $\phi$ 's $\in \mathbb{P}_{N}$. Then (2.16), expressed in terms of the corresponding $N+1$ vectors, reads $\left\langle D_{Q} u_{N}, W D_{Q} \phi\right\rangle=\left\langle\vee_{N}, W \phi\right\rangle$, and hence, $\vee_{N}=W^{-1} D_{Q}^{T} W D_{Q} u_{N}$. Actually, we have $D_{Q}=L_{D} \tilde{Q} L^{T} W$, where $\tilde{Q}:=\operatorname{diag}\left(\hat{q}_{0}\left\|L_{0}\right\|_{N}^{-2}, \ldots, \hat{q}_{N}\left\|L_{N}\right\|_{N}^{-2}\right)$ and $L, L_{D}$ are the $(N+1) \times(N+1)$ matrices with the elements

$$
(L)_{j k}=L_{k}\left(\xi_{j}\right), \quad\left(L_{D}\right)_{j k}=\left(\partial_{x} L_{k}\right)\left(\xi_{j}\right), \quad j, k=0,1, \ldots, N .
$$

Thus, by denoting $\tilde{D}:=\tilde{Q} L_{D}^{T} W L_{D} \tilde{Q}, \vee_{N}=L \tilde{D} L^{T} W u_{N}$. Since, for $0 \leq k \leq l \leq N$, we have from (2.6) that

$$
\left(L_{D}^{T} W L_{D}\right)_{k l}=\left(\partial_{x} L_{l}, \partial_{x} L_{k}\right)=\left.\left(L_{l} \partial_{x} L_{k}\right)\right|_{x=-1} ^{x=1}=\frac{1}{2}\left[1+(-1)^{k+l}\right] k(k+1),
$$


it follows that

$$
\begin{aligned}
(\tilde{D})_{l k}=(\tilde{D})_{k l}=\tilde{Q}_{k k} \tilde{Q}_{l l}\left(\partial_{x} L_{l}, \partial_{x} L_{k}\right) \\
\quad= \begin{cases}k(k+1) \gamma_{k} \gamma_{l} \hat{q}_{k} \hat{q}_{l}, & m<k \leq l \leq N, k+l \text { even, } \\
0 & \text { otherwise, }\end{cases}
\end{aligned}
$$

where $\gamma_{k}:=\left\|L_{k}\right\|_{N}^{-2}=(k+1 / 2)$ for $0 \leq k<N$ and $\gamma_{N}=N / 2$. Another way to reach this expression is to put $\phi=L_{k}$ in (2.16) so that, for $u_{N}(x)=\sum_{l=0}^{N} \hat{u}_{l} L_{l}(x)$,

$$
\begin{aligned}
\vee_{N}(x) & =\sum_{k=0}^{N} \frac{\left(\partial_{x}\left(Q u_{N}\right), \partial_{x}\left(Q L_{k}\right)\right)}{\left\|L_{k}\right\|_{N}^{2}} L_{k}(x)=\sum_{k=0}^{N} \sum_{l=0}^{N} \frac{\left(\hat{q}_{l} \partial_{x} L_{l}, \hat{q}_{k} \partial_{x} L_{k}\right)}{\left\|L_{k}\right\|_{N}^{2}} \hat{u}_{l} L_{k}(x) \\
& =\sum_{k=0}^{N} \sum_{l=0}^{N} \tilde{Q}_{k k} \tilde{Q}_{l l}\left(\partial_{x} L_{l}, \partial_{x} L_{k}\right)\left\|L_{l}\right\|_{N}^{2} \hat{u}_{l} L_{k}(x)=\sum_{k=0}^{N} \sum_{l=0}^{N}(\tilde{D})_{k l}\left\|L_{l}\right\|_{N}^{2} \hat{u}_{l} L_{k}(x) \\
& =\left(L_{0}(x), L_{1}(x), \ldots, L_{N}(x)\right) \tilde{D} L^{T} W\left(u_{N}\left(\xi_{0}\right), u_{N}\left(\xi_{1}\right), \ldots, u_{N}\left(\xi_{N}\right)\right)^{T} .
\end{aligned}
$$

Remark. To gain better insight into the SV operator we observe that the SV operator $Q$ is self-adjoint with respect to the discrete inner product $(\cdot, \cdot)$ and, thanks to (2.8), one can integrate by parts. Consequently, the SV expression on the left of (2.14) takes the form

$$
\varepsilon_{N}\left(\partial_{x}\left(Q u_{N}\right), \partial_{x}(Q \phi)\right)_{N}=\left.\varepsilon_{N} \partial_{x}\left(Q u_{N}\right) \cdot Q \phi\right|_{x=-1} ^{x=1}-\varepsilon_{N}\left(Q \partial_{x x}^{2}\left(Q u_{N}\right), \phi\right)_{N} .
$$

The realization of the SV operator here shows that $\vee_{N}$ is an approximation to $Q \partial_{x x}^{2}\left(Q u_{N}\right)$ which takes into account the boundary terms, thus preventing spurious boundary layers. Specifically, comparing (2.16) to (2.18), with $\phi=\phi_{i}, \phi_{i}\left(\xi_{j}\right)=\delta_{i j}$, yields

$$
\vee\left(\xi_{i}\right)=-Q \partial_{x x}^{2}\left(Q u_{N}\right)\left(\xi_{i}\right)+\left.\partial_{x} Q u_{N} \cdot Q \phi_{i}\right|_{-1} ^{1} .
$$

The SV operator here is different than the original SV method introduced in [23].

Let us "test" (2.14) against $\phi=\phi_{i}$, where $\phi_{i}$ is the standard characteristic polynomial of $\mathbb{P}_{N}(\Lambda)$ satisfying $\phi_{i}\left(\xi_{j}\right)=\delta_{i j}, 0 \leq i, j \leq N$. At the interior points we obtain

$$
\frac{d}{d t} u_{N}\left(\xi_{i}, t\right)+\frac{\partial}{\partial x} \mathcal{I}_{N} f\left(u_{N}\right)\left(\xi_{i}, t\right)=-\varepsilon_{N} \vee_{N}\left(\xi_{i}, t\right), \quad 1 \leq i \leq N-1
$$

For the boundary treatment we consider, for example, the case of an outflow boundary at $x=+1$ and an inflow boundary at $x=-1$. In this case $B\left(u_{N}(t)\right)=\lambda(t)(1-$ $x) \partial_{x} L_{N}(x)$. The value of $\lambda(t)$ is dictated by the prescribed inflow boundary data, setting $x=-1$ at (2.14). At the outflow boundary, $x=+1$, the boundary term $B\left(u_{N}(t)\right)$ vanishes and $(2.14)$ realizes the equation the same way it was discretized at the interior points in (2.19), namely,

$$
\frac{d}{d t} u_{N}(+1, t)+\frac{\partial}{\partial x} \mathcal{I}_{N} f\left(u_{N}\right)(+1, t)=-\varepsilon_{N} \vee_{N}(+1, t)
$$

We note that the last term on the right of (2.20) defined via (2.16) prevents the creation of a boundary layer. Equations (2.19)-(2.20), together with the inflow data prescribed at $x=-1, u_{N}(-1, t)=g_{-}(t)$, furnish a complete equivalent statement of the pseudospectral (collocation) viscosity approximation (2.14). 
3. Convergence of the SV method. To establish the necessary a priori estimates for $u_{N}$, we first prepare the following lemma.

Lemma 3.1. Consider the $S V$ operator $Q=Q_{m},(2.12)$ with the parameterization in (2.13). Then for any $\phi \in \mathbb{P}_{N}$,

$$
\begin{aligned}
& \left\|\partial_{x} \phi\right\|^{2} \leq 2\left\|\partial_{x}(Q \phi)\right\|^{2}+c m^{4} \ln N\|\phi\|^{2} \\
& \left\|\partial_{x}(Q \phi)\right\|^{2} \leq 2\left\|\partial_{x} \phi\right\|^{2}+c m^{4} \ln N\|\phi\|^{2} .
\end{aligned}
$$

Remark. The lemma shows the equivalence of the $H^{1}$ norm before and after application of the SV operator, $Q=Q_{m}$ for moderate size of $m_{N}<<N^{1 / 4}$. This holds despite the fact that for $m=m_{N} \sim c N^{\beta} \uparrow \infty$, the corresponding SV operator, $Q_{m}$ is spectrally small.

Proof. Let $\hat{\phi}_{l}$ be the coefficients of the Legendre expansion of $\phi(x)$, and

$$
J_{l, N}=\{j \mid l+1 \leq j \leq N, l+j \text { odd }\} .
$$

Then by the relation between the coefficients of the Legendre expansions of $\phi(x)$ and those for $\partial_{x} \phi(x)($ see [4]),

$$
\partial_{x} \phi(x)=\sum_{l=0}^{N-1} \hat{\phi}_{l}^{(1)} L_{l}(x), \quad \hat{\phi}_{l}^{(1)}=(2 l+1) \sum_{j \in J_{l, N}} \hat{\phi}_{j} .
$$

Next set $\hat{r}_{l}=1-\hat{q}_{l}$, and let $R$ denote the corresponding low modes filter

$$
R \phi(x):=\sum_{l=0}^{N} \hat{r}_{l} \hat{\phi}_{l} L_{l}(x) .
$$

Clearly $\hat{r}_{l}=1$ for $l \leq m$, and $\hat{r}_{l} \leq m^{2} l^{-2}$ for $l>m$. Since $\partial_{x} \phi(x) \equiv \partial_{x}(Q \phi(x))+$ $\partial_{x}(R \phi(x))$, it suffices to prove that

$$
\left\|\partial_{x}(R \phi)\right\|^{2} \leq c m^{4} \ln N\|\phi\|^{2} .
$$

We decompose $\partial_{x}(R \phi(x))=A_{1}(x)+A_{2}(x)$, where

$$
A_{1}(x):=\partial_{x}\left(\sum_{l=0}^{m} \hat{r}_{l} \hat{\phi}_{l} L_{l}(x)\right), \quad A_{2}(x):=\partial_{x}\left(\sum_{l=m+1}^{N} \hat{r}_{l} \hat{\phi}_{l} L_{l}(x)\right) .
$$

By standard inverse inequality (e.g., see [4]), $\left\|\partial_{x} \phi\right\| \leq c N^{2}\|\phi\| \forall \phi(x) \in \mathbb{P}_{N}$, and hence $\left\|A_{1}\right\|^{2} \leq c m^{4}\|\phi\|^{2}$. Further let $J_{l, N, m}=\left\{j \mid j \in J_{l, N}, j>m\right\}$. Then

$$
\begin{aligned}
\left\|A_{2}\right\|^{2} & =\sum_{l=0}^{N-1}(2 l+1)^{2}\left(\sum_{j \in J_{l, N, m}} \hat{r}_{j} \hat{\phi}_{j}\right)^{2}\left\|L_{l}\right\|^{2} \\
& \leq 2 \sum_{l=0}^{N-1}(2 l+1)\left(\sum_{j \in J_{l, N, m}}\left|\hat{r}_{j}\right|^{2}\left\|L_{j}\right\|^{-2}\right)\left(\sum_{j \in J_{l, N, m}}\left|\hat{\phi}_{j}\right|^{2}\left\|L_{j}\right\|^{2}\right) \\
& \leq c m^{4}\|\phi\|^{2} \sum_{l=0}^{N-1}(2 l+1) \sum_{j \in J_{l, N, m}} j^{-3} \\
& \leq c m^{4}\|\phi\|^{2}\left(m^{-2} \sum_{l=0}^{m}(2 l+1)+\sum_{l=m+1}^{N-1}(2 l+1) l^{-2}\right) \\
& \leq c m^{4} \ln N\|\phi\|^{2},
\end{aligned}
$$


and the desired estimates follow.

The following lemma is the heart of the matter.

Lemma 3.2. Consider the $S V$ scheme (2.14) with $H^{1}[0, T]$ boundary values, (2.2), and $H^{1}$ initial conditions, (2.3). Assume that the $S V$ solution remains uniformly bounded,

$$
\max _{0 \leq t \leq T}\left\|u_{N}(\cdot, t)\right\|_{L^{\infty}} \leq A_{\infty} .
$$

Then there exists a constant (depending on $A_{\infty}$ ) such that the following $H^{1}$-bound holds:

$$
\varepsilon_{N}\left[\left\|\partial_{t} u_{N}\right\|_{L^{2}\left([0, T], L^{2}(\Lambda)\right)}^{2}+\left\|\partial_{x} u_{N}\right\|_{L^{2}\left([0, T], L^{2}(\Lambda)\right)}^{2}\right] \leq \text { const. }
$$

Proof. To simplify the presentation, we shall deal with the prototype case where one boundary, say, $x=-1$, is an inflow boundary, while $x=1$ is an outflow one. Then

$$
B\left(u_{N}(t)\right)=\lambda(t)(1-x) \partial_{x} L_{N}(x) .
$$

Recall that $\xi_{j}$ are the zeros of $\partial_{x} L_{N}(x), 1 \leq j \leq N-1$ so that the boundary operator $B\left(u_{N}\right)$ vanishes at all but the inflow boundary point $x=-1$, where it involves the corresponding values of $\omega^{(0)}=2 / N(N+1)$ and $\partial_{x} L_{N}(-1)=(-1)^{N+1} N(N+1) / 2$. Thus

$$
\left(B\left(u_{N}(t)\right), v\right)_{N}=2(-1)^{N+1} \lambda(t) v(-1, t) .
$$

Let $\phi \equiv 1$ in (2.14). Since $I_{N} f\left(u_{N}(x, t)\right) \in \mathbb{P}_{N}$, we deduce, in view of $(2.8)$, that

$$
\partial_{t}\left(u_{N}(t), 1\right)+f\left(u_{N}(1, t)\right)-f\left(u_{N}(-1, t)\right)=2(-1)^{N+1} \lambda(t) .
$$

Consequently,

$$
|\lambda(t)| \leq \frac{1}{\sqrt{2}}\left\|\partial_{t} u_{N}(t)\right\|+\max \left(\left|f\left(u_{N}(1, t)\right)\right|,\left|f\left(u_{N}(-1, t)\right)\right|\right) .
$$

Further, set $\eta(t):=\int_{0}^{t} \lambda(s) d s$; then, integration of (3.3) yields for $t \leq T$

$$
|\eta(t)| \leq \frac{1}{\sqrt{2}}\left\|u_{N}(t)\right\|+\frac{1}{\sqrt{2}}\left\|u_{N}(0)\right\|+t \max _{|z| \leq A}|f(z)|, \quad \eta(t):=\int_{0}^{t} \lambda(s) d s .
$$

Next we recall the SV parameterization in (2.15), $\varepsilon_{N} \sim c N^{-\alpha}, \quad m \sim c N^{\beta}$, $0<4 \beta<\alpha \leq 1$. To get the desired $H^{1}$-energy bound, we integrate the SV scheme against $u_{N}$. That is, we set $\phi=u_{N}$ in (2.14). Let $F(u)=\int^{u} w f^{\prime}(w) d w$ denote entropy flux corresponding to the quadratic entropy, $U(u)=u^{2} / 2$. Using (2.11) followed by Lemma 3.1 we find

$$
\begin{aligned}
& \frac{1}{2} \frac{d}{d t}\left\|u_{N}(t)\right\|_{N}^{2}+F\left(u_{N}(1, t)\right)-F\left(u_{N}(-1, t)\right) \\
& +\varepsilon_{N}\left(\partial_{x}\left(Q u_{N}(t)\right), \partial_{x}\left(Q u_{N}(t)\right)\right)+2(-1)^{N} \lambda(t) u_{N}(-1, t) \\
& =\left(\partial_{x} f\left(u_{N}(t)\right), u_{N}(t)\right)-\left(\partial_{x} I_{N} f\left(u_{N}(t)\right), u_{N}(t)\right)_{N} \\
& =-\left(\left(I-I_{N}\right) f\left(u_{N}(t)\right), \partial_{x} u_{N}(t)\right) \\
& \leq \frac{c}{N}\left\|\partial_{x} f\left(u_{N}(t)\right)\right\| \cdot\left\|\partial_{x} u_{N}(t)\right\| \leq \frac{c_{A}}{N}\left(\left\|\partial_{x}\left(Q u_{N}(t)\right)\right\|^{2}+m^{4} \ln N\left\|u_{N}(t)\right\|_{N}^{2}\right) .
\end{aligned}
$$


Here and below, $c_{A}$ stand for various constants depending on the uniform bound $A_{\infty}:=\max \left\|u_{N}\right\|_{L^{\infty}}$. Thus for any $t \leq T$,

$$
\begin{aligned}
& \left\|u_{N}(t)\right\|_{N}^{2}+2\left(\varepsilon_{N}-\frac{c_{A}}{N}\right) \int_{0}^{t}\left\|\partial_{x}\left(Q u_{N}(s)\right)\right\|^{2} d s \\
& \leq c_{0}+2 \int_{0}^{t}\left(\frac{c_{A} m^{4} \ln N}{N}\left\|u_{N}(s)\right\|^{2}+2 \max _{|z| \leq A}|F(z)|-2(-1)^{N} \lambda(s) g_{-}(s)\right) d s .
\end{aligned}
$$

By (3.5),

$$
\begin{aligned}
\left|\int_{0}^{t} \lambda(s) g_{-}(s) d s\right| & =\left|g_{-}(t) \eta(t)-g_{-}(0) \eta(0)-\int_{0}^{t} g_{-}^{\prime}(s) \eta(s) d s\right| \\
& \leq\left|g_{-}(t)\right|\left(\left\|u_{N}(t)\right\|+c_{A}+c_{0}\right)+\int_{0}^{t}\left|g_{-}^{\prime}(s)\right|\left(\left\|u_{N}(s)\right\|+c_{A}+c_{0}\right) d s .
\end{aligned}
$$

Putting together the two estimates above, we have from (3.1) and the SV parameterization in (2.15) that

$$
\left\|u_{N}(t)\right\|^{2}+\varepsilon_{N} \int_{0}^{t}\left\|\partial_{x}\left(Q u_{N}(s)\right)\right\|^{2} d s \leq c_{A}\left(\left\|g_{-}\right\|_{H^{1}(0, t)}^{2}+t+1\right)+c_{0} .
$$

Using Lemma 3.1 and (2.15) again, we arrive at

$$
\varepsilon_{N}\left\|\partial_{x} u_{N}\right\|_{L^{2}\left([0, T] ; L^{2}(\Lambda)\right)}^{2} \leq c_{A}\left(\|g\|_{H^{1}(0, T)}^{2}+T+1\right)+c_{0} .
$$

Next, we set $\phi=\partial_{t} u_{N}$ in the SV weak formulation (2.14). By (2.9), (2.7), and (3.4),

$$
\left\|\partial_{t} u_{N}(t)\right\|^{2}+\frac{\varepsilon_{N}}{2} \frac{d}{d t}\left\|\partial_{x}\left(Q u_{N}(t)\right)\right\|^{2} \leq c_{A}\left\|\partial_{x} u_{N}(t)\right\|^{2}+\frac{1}{2}\left\|\partial_{t} u_{N}(t)\right\|^{2}+c_{A}\left|\frac{d}{d t} g_{-}(t)\right|^{2}+c_{A} .
$$

Temporal integration of the above inequality followed by (3.6) implies

$$
\begin{gathered}
\left\|\partial_{t} u_{N}\right\|_{L^{2}\left([0, T] ; L^{2}(\Lambda)\right)}^{2} \leq c_{A}\left(\left\|\partial_{x} u_{N}\right\|_{L^{2}\left((0, T) ; L^{2}(\Lambda)\right)}^{2}+\left\|g_{-}\right\|_{H^{1}(0, T)}^{2}+1\right) \\
\leq \frac{c_{A}}{\varepsilon_{N}}\left(\left\|g_{-}\right\|_{H^{1}(0, T)}^{2}+T+c_{0}+1\right) .
\end{gathered}
$$

Inequalities (3.6) and (3.7) conclude the proof.

Equipped with the $H^{1}$-bound and Lemma 3.2 we are now ready to state the main stability result of this paper.

TheOREM 3.3. Let $u_{N}$ be the solution of the SV scheme (2.14)-(2.15). Assume that it remains uniformly bounded so that (3.1) holds. Then $u_{N}$ tends (strongly in $\left.L_{\mathrm{loc}}^{p}(Q) 1 \leq p<\infty\right)$ to a weak solution, $u$, of the initial-boundary value problem $(2.1)$.

If, in addition, the $S V$ amplitude is set $\varepsilon_{N} \sim N^{-\alpha}$ with $\alpha<1$, then $u$ is the unique entropy solution.

Remark. The uniform bound of the one-dimensional Fourier SV solution was proved in $[23,32]$. For the corresponding multidimensional proof we refer to [6]. One might expect a proof of the assumed uniform bound (3.1) to be carried out for the current Legendre case along similar lines, but it would require more technical arguments which are beyond the scope of this paper. 
Proof. Let $\Omega=\Lambda \times[0, T]$ and define $(\cdot, \cdot)_{\Omega}$ and $\|\cdot\|_{\Omega}$ as before. Let

$$
(v, w)_{\Omega, N}=\int_{0}^{T}(v(t), w(t))_{N} d t .
$$

We still use $c_{A}$ to denote, as before, a constant depending on $A_{\infty}$ with possible dependence on $T$.

We want to show that the entropy production of $u_{N}$ is compact in $H_{\text {loc }}^{-1}(\Omega)$. To this end, we consider, for an arbitrary convex entropy pair $(U, F)$,

$$
\left(\partial_{t} U\left(u_{N}\right)+\partial_{x} F\left(u_{N}\right), w\right)_{\Omega} \equiv \sum_{j=1}^{5} G_{j}\left(U^{\prime}\left(u_{N}\right) \phi\right) .
$$

We decompose the entropy production to the five terms on the right given by

$$
\begin{aligned}
& G_{1}(\psi)=\left(\partial_{t} u_{N}+\partial_{x} f\left(u_{N}\right), \psi-\psi_{N}\right)_{\Omega}, \\
& G_{2}(\psi)=\left(\partial_{x} f\left(u_{N}\right)-\partial_{x} I_{N} f\left(u_{N}\right), \psi_{N}\right)_{\Omega}, \\
& G_{3}(\psi)=\left(\partial_{t} u_{N}+\partial_{x} I_{N} f\left(u_{N}\right), \psi_{N}\right)_{\Omega}-\left(\partial_{t} u_{N}+\partial_{x} I_{N} f\left(u_{N}\right), \psi_{N}\right)_{\Omega, N}, \\
& G_{4}(\psi)=\varepsilon_{N}\left(\partial_{x}\left(R u_{N}\right), \partial_{x} \psi_{N}\right)_{\Omega}+\varepsilon_{N}\left(\partial_{x} u_{N}, \partial_{x}\left(R \psi_{N}\right)\right)_{\Omega}, \\
& G_{5}(\psi)=-\varepsilon_{N}\left(\partial_{x} u_{N}, \partial_{x} \psi_{N}\right)_{\Omega}-\varepsilon_{N}\left(\partial_{x}\left(R u_{N}\right), \partial_{x}\left(R \psi_{N}\right)\right)_{\Omega} .
\end{aligned}
$$

The last identity holds for arbitrary $\psi_{N} \in \mathbb{P}_{N}$. Following Maday, Ould Kaber, and Tadmor [23, section 5] we specify

$$
\psi_{N}=\mathcal{J}_{N} \psi:=\int_{-1}^{x} P_{N-1} \partial_{y} \psi(y, t) d y .
$$

This specific choice will play an essential role in the derivation of the entropy condition below. Observe that $\psi_{N} \in \mathbb{P}_{N}$ with $\psi_{N}(-1, t)=0$. We recall that the operator $R$ above denotes the complement operator, $Q+R=I d$, associated with symbols $\hat{r}_{l}=1-\hat{q}_{l}$. We proceed with the upper bound on the five terms on the right of (3.8).

By (2.10), (3.6), and (3.7),

$$
\left|G_{1}(\psi)\right| \leq \frac{c_{A}}{\sqrt{\varepsilon_{N}}}\left\|\psi-\psi_{N}\right\|_{D} \leq \frac{c_{A}}{N \sqrt{\varepsilon_{N}}}\left\|\partial_{x} \psi\right\|_{\Omega} .
$$

According to (3.6),

$\left|G_{2}(\psi)\right|=\left|\left(f\left(u_{N}\right)-I_{N} f\left(u_{N}\right), \partial_{x} \psi_{N}\right)_{\Omega}\right| \leq \frac{c}{N}\left\|\partial_{x} f\left(u_{N}\right)\right\|_{\Omega}\left\|\partial_{x} \psi_{N}\right\|_{\Omega} \leq \frac{c_{A}}{N \sqrt{\varepsilon_{N}}}\left\|\partial_{x} \psi_{N}\right\|_{\Omega}$.

By virtue of (2.11) and (3.7),

$$
\left|G_{3}(\psi)\right|=\left|\left(\partial_{t} u_{N}, \psi_{N}\right)_{\Omega}-\left(\partial_{t} u_{N}, \psi_{N}\right)_{\Omega, N}\right| \leq \frac{c_{A}}{N}\left\|\partial_{t} u_{N}\right\|_{\Omega}\left\|\partial_{x} \psi_{N}\right\|_{\psi} \leq \frac{c_{A}}{N \sqrt{\varepsilon_{N}}}\left\|\partial_{x} \psi_{N}\right\|_{\Omega}
$$

To proceed, we utilize Lemma 3.1, $\left\|\partial_{x}(R \phi)\right\|_{\Omega} \leq c m^{2} \sqrt{\ln N}\|\phi\|_{\Omega}$. Applying this with $\phi=u_{N}, \psi_{N}$ we find

$$
\begin{aligned}
& \left|G_{4}(\psi)\right| \leq \varepsilon_{N} c m^{2} \sqrt{\ln N}\left\|u_{N}\right\|_{\Omega}\left\|\partial_{x} \psi_{N}\right\|_{\Omega}+\varepsilon_{N}\left\|\partial_{x} u_{N}\right\|_{\psi}\left\|\partial_{x}\left(R \psi_{N}\right)\right\|_{\Omega} \\
& \leq c_{A} m^{2} \sqrt{\ln N}\left(\varepsilon_{N}\left\|\partial_{x} \psi_{N}\right\|_{\Omega}+\sqrt{\varepsilon_{N}}\left\|\psi_{N}\right\|_{\Omega}\right) .
\end{aligned}
$$


Similarly

$$
\left|G_{5}(\psi)\right| \leq \sqrt{\varepsilon_{N}} c_{A}\left\|\partial_{x} \psi_{N}\right\|_{\Omega}+\varepsilon_{N} m^{4} \ln N\left\|\psi_{N}\right\|_{\Omega} .
$$

The previous statements, with $\psi=U^{\prime}\left(u_{N}\right) \phi$, tell us that

$$
\begin{aligned}
& \left|\sum_{j=1}^{5} G_{j}\left(U^{\prime}\left(u_{N}\right) \phi\right)\right| \\
\leq & c_{A}\left(\frac{1}{N \sqrt{\varepsilon_{N}}}+\varepsilon_{N} m^{2} \sqrt{\ln N}+\sqrt{\varepsilon_{N}}\right)\left(\left\|\partial_{x} u_{N}\right\|_{\Omega}\|\phi\|_{L^{\infty}(\Omega)}+c_{A}\left\|\partial_{x} \phi\right\|_{\Omega}\right) \\
\leq & c_{A}\left(\|\phi\|_{L^{\infty}(\Omega)}+\left(\frac{1}{N \sqrt{\varepsilon_{N}}}+\varepsilon_{N} m^{2} \sqrt{\ln N}+\sqrt{\varepsilon_{N}}\right)\left\|\partial_{x} \phi\right\|_{\Omega}\right) .
\end{aligned}
$$

Thus, the entropy production $\partial_{t} U\left(u_{N}\right)+\partial_{x} F\left(u_{N}\right)$ can be written as a sum of two terms - the first tends to zero in $H^{-1}(\Omega)$ and the second is bounded in $L^{1}(\Omega)$. In view of the Murat lemma [5], bounded sequences in $W^{-1, p}\left(\mathbb{R}^{N}\right) \cap L^{1}\left(\mathbb{R}^{N}\right), 2<p<\infty$ form a compact subset of $H^{-1}(\Omega)$. We conclude that the entropy production of the SV solution is $H^{-1}$-compact which, in turn, by compensated compactness arguments [34], implies that $u_{N}$ converges strongly (in $L_{\mathrm{loc}}^{p}\left(\Lambda \times \mathbb{R}_{t}^{+}\right), p<\infty$ ) to a weak solution, $u$, of the conservation law (2.1).

It remains to show that $u$ is indeed the unique entropy solution. To verify the entropy condition for $\varepsilon_{N} \sim c N^{-\alpha}, \alpha<1$, we proceed as follows. It is easy to see that

$$
\begin{aligned}
\sum_{j=1}^{3}\left|G_{j}\left(U^{\prime}\left(u_{N}\right) \phi\right)\right| & \leq \frac{c_{A}}{N \sqrt{\varepsilon_{N}}}\left\|\partial_{x}\left(U^{\prime}\left(u_{N}\right) \phi\right)_{N}\right\|_{\Omega} \\
& \leq \frac{c_{A}}{N \sqrt{\varepsilon_{N}}}\left(\left\|\partial_{x} u_{N}\right\|_{\Omega}\|\phi\|_{L^{\infty}(\Omega)}+\left\|u_{N}\right\|_{L^{\infty}(\Omega)}\left\|\partial_{x} \phi\right\|_{\Omega}\right) \\
& \leq c_{A}\left(N^{\alpha-1}\|\phi\|_{L^{\infty}(\Omega)}+N^{\frac{\alpha}{2}-1}\left\|\partial_{x} \phi\right\|_{\Omega}\right) \longrightarrow 0
\end{aligned}
$$

By the choice of the SV parameters in (2.15), together with (3.6) and (3.10), we have

$$
\begin{aligned}
\left|G_{4}\left(U^{\prime}\left(u_{N}\right) \phi\right)\right| & \leq c_{A} \varepsilon_{N} m^{2} \sqrt{\ln N}\left\|\partial_{x}\left(U^{\prime}\left(u_{N}\right) w\right)\right\|_{\Omega}+c_{A} \sqrt{\varepsilon_{N}} m^{2} \sqrt{\ln N}\left\|U^{\prime}\left(u_{N}\right) \phi\right\|_{\Omega} \\
& \leq c_{A} \sqrt{\varepsilon} M^{2} \sqrt{\ln N}\left(\|\phi\|_{\Omega}+\left\|\partial_{x} \phi\right\|_{\Omega}\right) \longrightarrow 0 .
\end{aligned}
$$

Finally, let $\left(U^{\prime}\left(u_{N}\right) \phi\right)_{N}$ denote our usual projection, $\left(U^{\prime}\left(u_{N}\right) \phi\right)_{N}=\mathcal{J}_{N}\left(U^{\prime}\left(u_{N}\right) \phi\right.$. It is here that we take advantage of our special choice of projection, $\mathcal{J}_{N}$. Indeed, for any nonnegative test function, $\phi(x) \geq 0$, we find

$$
\begin{aligned}
G_{5}\left(U^{\prime}\left(u_{N}\right) \phi\right)= & -\varepsilon\left(\partial_{x} u_{N}, P_{N-1} \partial_{x}\left(U^{\prime}\left(u_{N}\right) \phi\right)\right)_{\Omega}-\varepsilon_{N}\left(\partial_{x}\left(R u_{N}\right), \partial_{x}\left(R\left(U^{\prime}\left(u_{N}\right) \phi\right)_{N}\right)\right)_{\Omega} \\
= & -\varepsilon_{N}\left(\partial_{x} u_{N}, U^{\prime \prime}\left(u_{N}\right) w \partial_{x} u_{N}\right)_{\Omega}-\varepsilon_{N}\left(\partial_{x} u_{N}, U^{\prime}\left(u_{N}\right) \partial_{x} w\right)_{\Omega} \\
& -\varepsilon_{N}\left(\partial_{x}\left(R u_{N}\right), \partial_{x}\left(R\left(U^{\prime}\left(u_{N}\right) \phi\right)_{N}\right)\right)_{\Omega} \\
\leq & -\varepsilon_{N}\left(\partial_{x} u_{N}, U^{\prime}\left(u_{N}\right) \partial_{x} \phi\right)_{\Omega}-\varepsilon_{N}\left(\partial_{x}\left(R u_{N}\right), \partial_{x}\left(R\left(U^{\prime}\left(u_{N}\right) \phi\right)_{N}\right)\right)_{\Omega} \\
\leq & c_{A} \sqrt{\varepsilon_{N}}\left\|\partial_{x} \phi\right\|_{\Omega}+c_{A} \varepsilon_{N} m^{4} \ln N\|\phi\|_{\Omega} \longrightarrow 0 .
\end{aligned}
$$

It follows that $u$ satisfies the entropy inequality (2.4) in the sense of distribution, and so it is the unique entropy solution.

Remark. Maday, Ould Kaber, and Tadmor [23] introduced the nonperiodic SV scheme with SV of the form

$$
\varepsilon\left(Q \partial_{x} u_{N}(t), \partial_{x} \phi\right)_{N}
$$


parameterized with viscosity coefficients [23, equation (2.2)]

$$
\begin{cases}\hat{q}_{l}=0 & \text { for } l \leq m, \\ \hat{q}_{l} \geq 1-\frac{m^{4}}{l^{4}} & \text { for } m<l \leq N .\end{cases}
$$

Observe that the SV operator is applied here once to the first derivative of the SV solution. Thus the "amount" of high-modes smoothing introduced in (3.11) is comparable to the amount of SV introduced here in (2.13) which is activated twice-before and after differentiation. The main difference between these two approaches, however, lies in the activation of high-modes diffusion to the SV solution rather than to its first derivative, as in (3.11).

In the proof of the main result of [23], Corollary 3.2 plays an important role, analogous to our Lemma 3.1. According to [23, Corollary 3.2], for any $\phi \in \mathbb{P}_{N}$, we have

$$
\left\|\partial_{x} \phi\right\|^{2} \leq\left\|\partial_{x} \phi\right\|_{Q}^{2}+c m^{4} \ln N\|\phi\|^{2},
$$

where $\left\|\partial_{x} \phi\right\|_{Q}^{2}$ stands for the weighted norm $\left\|\partial_{x} \phi\right\|_{Q}^{2}=\left(Q \partial_{x} \phi, \partial_{x} \phi\right)$. The argument, based on dyadic decomposition of $\phi$, fails, however, precisely because of the additional terms introduced by differentiation of each dyadic bloc. Indeed, let us take $\phi(x)=$ $L_{N}(x)$ so that

$$
\partial_{x} \phi(x)=\sum_{\substack{l=0 \\ l+N \text { odd }}}^{N-1}(2 l+1) L_{l}(x)
$$

and let $\hat{q}_{l}=1$ for $m<l \leq N$. Then on the one hand,

$$
\left\|\partial_{x} \phi\right\|^{2}-\left\|\partial_{x} \phi\right\|_{Q}^{2}=\sum_{\substack{l=0 \\ l+N \text { odd }}}^{m}(2 l+1)^{2}\left\|L_{l}\right\|^{2}=2 \sum_{\substack{l=0 \\ l+N \text { odd }}}^{m}(2 l+1)=O\left(m^{2}\right) ;
$$

on the other hand, however, $\|\phi\|^{2}=\left(N+\frac{1}{2}\right)^{-1}$. To establish the a priori estimates (3.6) and (3.7) therefore requires $m_{N}^{4} \leq c \ln N$ which is much stronger than the condition imposed in $[23,(2.15)]$. Thus the result of the present paper is a correction and an improvement of the result in [23].

4. Numerical results. In this section, we give some numerical results of the scheme (2.19)-(2.20). We consider the Hopf equation (or inviscid Burgers equation)

$$
\partial_{t} u(x, t)+\partial_{x} u^{2}(x, t) / 2=0, \quad(x, t) \in \Omega,
$$

with initial values

$$
u(0, x)=1+\frac{1}{2} \sin \pi x, \quad x \in[-1,1]
$$

and boundary conditions $u(-1, t)=g(t)$, where the inflow data are taken from the outflow boundary, i.e., $g(t)=u(1, t)$.

This is the example presented in Maday, Ould Kaber, and Tadmor [23]. We compute the same problem for the purpose of comparison. In Figure 4.1(a),(b), we show the numerical results of the Legendre viscosity method (2.14)-(2.15) with 
(a)

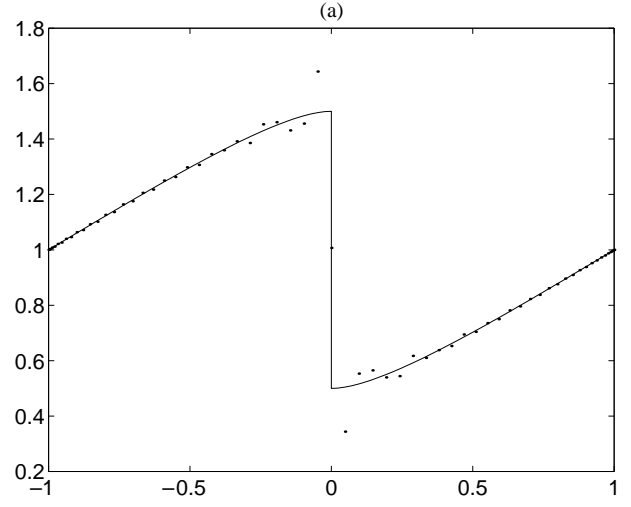

(b)

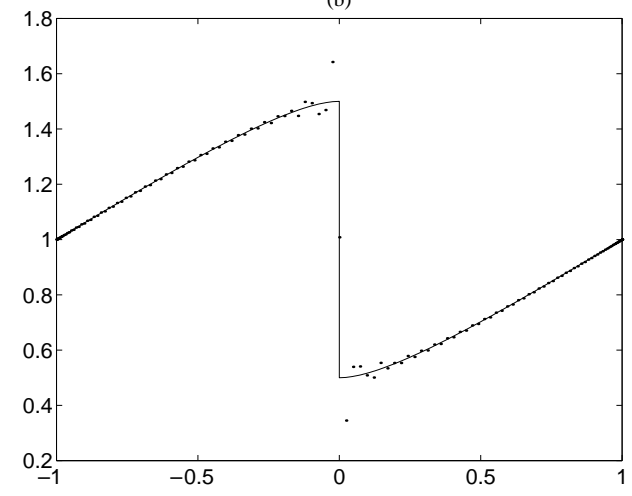

FIG. 4.1. Solution of the proposed pseudospectral viscosity method (2.14) with (a) $N=64$ modes on the left and (b) $N=128$ mode on the right.
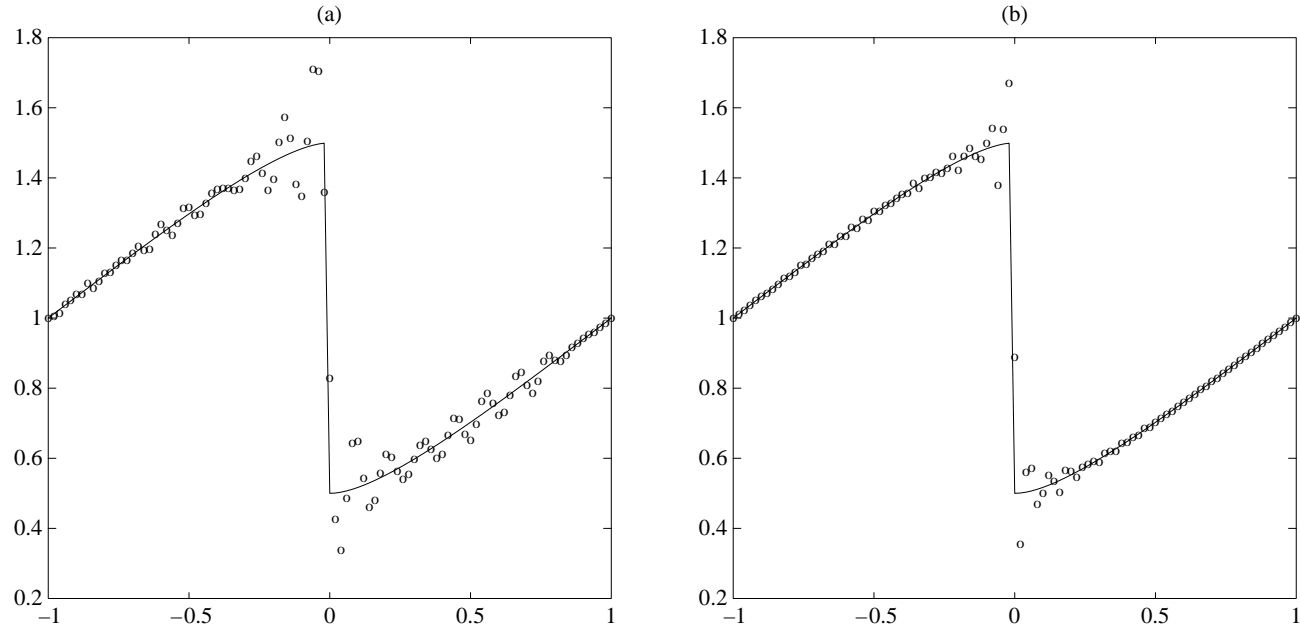

FIG. 4.2. The (preprocessed) pseudospectral viscosity solution of [23] based on $S V(3.10)-(3.11)$, with (a) $N=64$ modes and (b) $N=128$ modes.

$N=64$ and $N=128$ modes, respectively. The Legendre SV solution is computed with SV parameters $\left(\varepsilon=\varepsilon_{N}, m=m_{N}\right)$ taken as $\varepsilon \simeq N^{-1}$ and $m=m_{N} \simeq N^{1 / 4}$. For time discretization, we use the fourth-order Runge-Kutta scheme with time step $\Delta t=10^{-5}$. These should be compared with the corresponding results of [23] displayed in Figure 4.2.

To enhance the convergence up to the location of the shock (at $x=0$ ) the Legendre pseudospectral viscosity solution at time $t=1$ is postprocessed using the Gegenbauer reconstruction [12], where $u_{N}(\cdot, t=1)$ is expanded in terms of Gegenbauer polynomials $C_{n}^{\lambda}(x)$ for $0 \leq n \leq \ell_{N}$ in each of the smooth regions $[-1,0]$ and $[0,1]$. Figure 4.3(a),(b) shows the corresponding results after the postprocessing, which enjoy better resolution than those reported in [23, Figure 6.3].

The improved error decay of the postprocessed Legendre viscosity solution presented in Table 4.1 below is quantified in terms of the $L^{1}([-1,-0.2] \cup[0.2,1])$ norm (away from the jump). Gegenbauer postprocessing was implemented with $\lambda=\ell_{N} \simeq$ $0.05 N$. Compared with the preprocessed results in Table 4.1, column (a), the errors in 

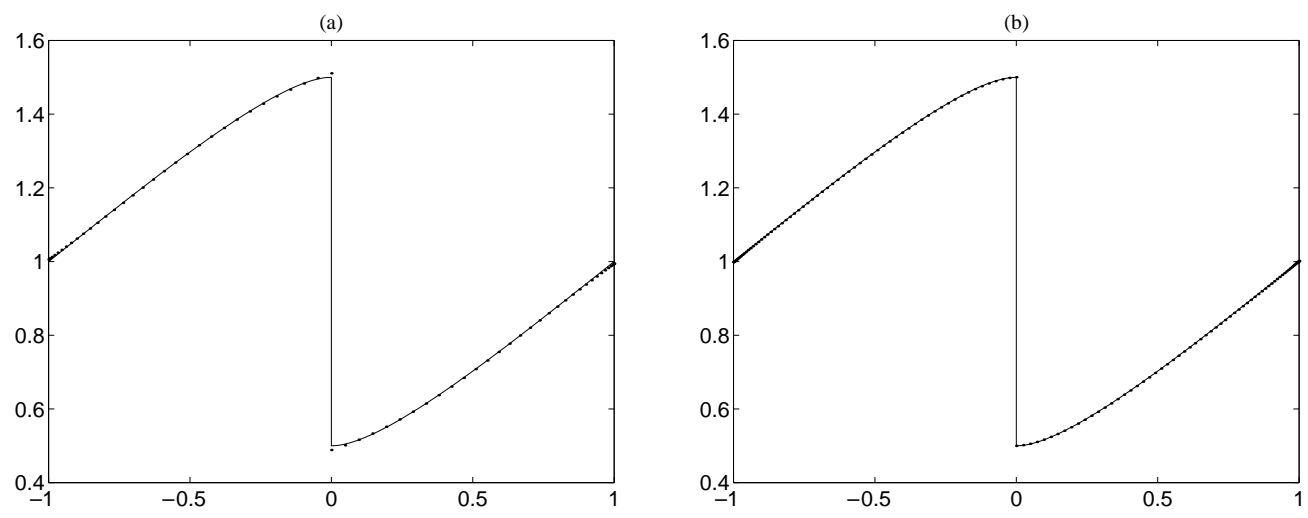

FIG. 4.3. The SV solution in Figure 4.1 after postprocessing.

TABLE 4.1

$L^{1}([-1,-0.2] \cup[0.2,1])$ error of the $S V$ method, (a) without postprocessing, (b) after Gegenbauer postprocessing.

\begin{tabular}{|r|c|c|}
\hline \multicolumn{1}{|c|}{$N$} & $(\mathrm{a})$ & $(\mathrm{b})$ \\
\hline 40 & $2.3464 \mathrm{e}-2$ & $3.3437 \mathrm{e}-4$ \\
\hline 80 & $1.1740 \mathrm{e}-2$ & $2.5697 \mathrm{e}-5$ \\
\hline 160 & $5.4598 \mathrm{e}-3$ & $6.8909 \mathrm{e}-6$ \\
\hline
\end{tabular}

the smooth part of the solution, Table 4.1, column (b), are considerably smaller after the postprocessing. Moreover, the convergence rate of the preprocessed results is linear; after postprocessing one recovers a considerably faster error decay with increasing $N$ (the error decay with $N=160$ hinges on the rather sensitive parameterization of the Gegenbauer postprocessing).

\section{REFERENCES}

[1] S. Abarbanel, D. Gottlieb, And E. Tadmor, Spectral methods for discontinuous problems, in Numerical Methods for Fluid Dynamics II, Proceedings of the 1985 Conference on Numerical Methods for Fluid Dynamics, K. W. Morton and M. J. Baines, eds., Clarendon Press, Oxford, 1986, pp. 129-153.

[2] O. Andreassen, I. Lie, and C.E. Wasberg, The spectral viscosity method applied to simulation of waves in a stratified atmosphere, J. Comput. Phys., 110 (1994), pp. 257-273.

[3] C. Bernardi and Y. Maday, Approximations Spectrales des Problèmes aux Limites Elliptiques, Springer-Verlag, Berlin, 1992.

[4] C. Canuto, M. Y. Hussaini, A. Quarteroni, and T. Zang, Spectral Method in Fluid Dynamics, Springer-Verlag, Berlin, 1988.

[5] G.-Q. Chen, The Compensated Compactness Method and the System of Isentropic Gas Dynamics, Preprint MSRI-00527-91, Mathematical Science Research Institute, Berkeley, CA, 1990.

[6] G.-Q. Chen, Q. Du, And E. Tadmor, Spectral viscosity approximations to multidimensional scalar conservation laws, Math. Comp., 61 (1993), pp. 629-643.

[7] A. Gelb and E. Tadmor, Detection of edges in spectral data, Appl. Comp. Harmonic Anal., 7 (1999), pp. 101-135.

[8] A. Gelb And E. Tadmor, Enhanced spectral viscosity approximations for conservation laws, Appl. Numer. Math., 33 (2000), pp. 3-21.

[9] A. Gelb and E. Tadmor, Detection of edges in spectral data II. Nonlinear enhancement, SIAM J. Numer. Anal., 38 (2000), pp. 1389-1408.

[10] D. Gottlieb L. Lustman, And E. TAdmor, Stability analysis of spectral methods for hyperbolic initial-boundary value systems, SIAM J. Numer. Anal., 24 (1987), pp. 241-256. 
[11] D. Gottlieb And S. A. Orszag, Numerical Analysis of Spectral Methods: Theory and Applications, SIAM, Philadelphia, 1977.

[12] D. Gottlieb And C.-W. Shu, On the Gibbs phenomenon and its resolution, SIAM Rev., 39 (1998), pp. 644-668.

[13] D. Gottlieb, C.-W. Shu, A. Solomonoff, and H. Vandeven, On the Gibbs phenomenon I: Recovering exponential accuracy from the Fourier partial sum of a nonperiodic analytic function, J. Comput. Appl. Math., 43 (1992), pp. 81-92.

[14] D. Gottlieb And E. TAdmor, Recovering pointwise values of discontinuous data within spectral accuracy, in Progress and Supercomputing in Computational Fluid Dynamics, Proceedings of a 1984 U.S.-Israel Workshop, Progr. Sci. Comput. 6, E. M. Murman and S. S. Abarbanel, eds., Birkhauser, Boston, 1985, pp. 357-375.

[15] B.-Y. Guo, Spectral Methods and Their Applications, World Scientific, Singapore, 1998.

[16] K. T. Joseph And P. G. LeFloch, Boundary layers in weak solutions to hyperbolic conservation laws, Arch. Rational Mech. Anal., 147 (1999), pp. 47-88.

[17] G.-S. Karamanos And G. Karniadakis, A spectral vanishing viscosity method for large eddy simulations, J. Comput. Phys., 163 (2000), pp. 22-50.

[18] P. D. Lax, Hyperbolic Systems of Conservation Laws and the Mathematical Theory of Shock Waves, CBMS-NSF Regional Conf. Ser. Appl. Math. 11, SIAM, Philadelphia, 1972.

[19] I. LIE, On the multidomain spectral viscosity method in multidomain Chebyshev discretizations, in Proceedings of the Third International Conference on Spectral and High-Order Methods (ICOSAHOM '95), A. V. Ilin and L. R. Scott, eds., Department of Mathematics, University of Houston, Houston, TX, 1996, pp. 121-130.

[20] H. MA, Chebyshev-Legendre spectral viscosity method for nonlinear conservation laws, SIAM J. Numer. Anal., 35 (1998), pp. 869-892.

[21] H. MA, Chebyshev-Legendre super spectral viscosity method for nonlinear conservation laws, SIAM J. Numer. Anal., 35 (1998), pp. 893-908.

[22] Y. MADAY AND E. TADMOR, Analysis of the spectral vanishing viscosity method for periodic conservation laws, SIAM J. Numer. Anal., 26 (1989), pp. 854-870.

[23] Y. MAday, S. M. Ould KABer, And E. TADMOR, Legendre pseudospectral viscosity method for nonlinear conservation laws, SIAM J. Numer. Anal., 30 (1993), pp. 321-342.

[24] A. Majda, J. McDonough, And S. Osher, The Fourier method for nonsmooth initial data, Math. Comp., 30 (1978), pp. 1041-1081.

[25] S. M. Ould Kaber, A Legendre pseudospectral viscosity method, J. Comp. Phys., 128 (1996), pp. $165-180$.

[26] S. SCHOCHET, The rate of convergence of spectral-viscosity methods for periodic scalar conservation laws, SIAM J. Numer. Anal., 27 (1990), pp. 1142-1159.

[27] C. W. Shu And P. Wong, A note on the accuracy of spectral method applied to nonlinear conservation laws, J. Sci. Comput., 10 (1995), pp. 357-369.

[28] J. SMOLLER, Shock Waves and Reaction-Diffusion Equations, Springer-Verlag, New York, 1983.

[29] E. TADMOR, Convergence of spectral methods for nonlinear conservation laws, SIAM J. Numer. Anal., 26 (1989), pp. 30-44.

[30] E. TADMOR, Shock capturing by the spectral viscosity method, Comput. Methods Appl. Mech. Engrg., 80 (1990), pp. 197-208.

[31] E. TADMOR, Local error estimates for discontinuous solutions of nonlinear hyperbolic equations, SIAM J. Numer. Anal., 28 (1991), pp. 891-906.

[32] E. TADMOR, Total-variation and error estimates for spectral viscosity approximations, Math. Comp., 60 (1993), pp. 245-256.

[33] E. TADMOR, Super viscosity and spectral approximations on nonlinear conservation laws, in Numerical Methods for Fluid Dynamics IV, Proceedings of the 1992 Conference on Numerical Methods for Fluid Dynamics, M. J. Baines and K. W. Morton, eds., Clarendon Press, Oxford, 1993, pp. 69-82.

[34] L. TARTAR, Compensated compactness and applications to partial differential equations, in Nonlinear Analysis and Mechanics: Heriot-Watt Symposium, Vol. IV, Res. Notes in Math. 39, J. Knopps, ed., Pitman, Boston, London, 1979, pp. 136-212. 